\section{STRUCTURAL} BIOLOGY

ISSN 2059-7983

\title{
The crystal structure of AjiA1 reveals a novel structural motion mechanism in the adenylate- forming enzyme family
}

\author{
Fernanda C. R. de Paiva, ${ }^{a}$ Karen Chan, ${ }^{\mathrm{b}}$ Markiyan Samborskyy, ${ }^{\mathrm{b}}$ Ariel M. Silber, \\ Peter F. Leadlay ${ }^{\mathrm{b}}$ and Marcio V. B. Dias ${ }^{\mathrm{a}, \mathrm{d}}$ *
}

Received 29 June 2020

Accepted 7 October 2020

Edited by P. Langan, Oak Ridge National Laboratory, USA

Keywords: adenylate-forming enzymes; AjiA1; ATP-dependent CoA ligase; benzoxazole antibiotics; loop swapping.

PDB reference: AjiA1, 6wuq

Supporting information: this article has supporting information at journals.iucr.org/d

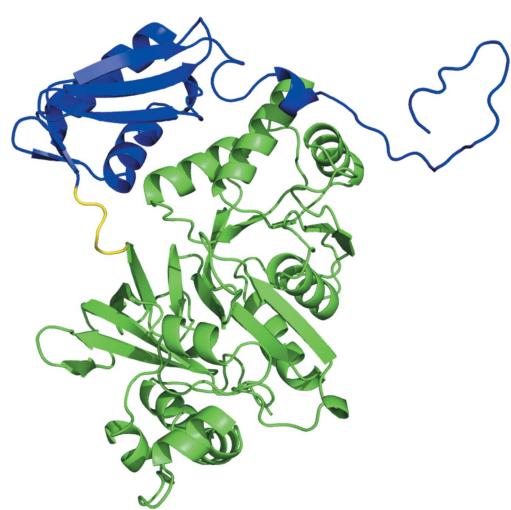

C 2020 International Union of Crystallography
aDepartment of Microbiology, Institute of Biomedical Sciences II, University of São Paulo, São Paulo, Brazil, 'b Department of Biochemistry, University of Cambridge, Cambridge, United Kingdom, 'LaBTryps - Laboratory of Biochemistry of Tryps, Department of Parasitology, Institute of Biomedical Sciences, University of São Paulo, São Paulo, Brazil, and ${ }^{\mathbf{d} D e p a r t m e n t}$ of Chemistry, University of Warwick, Coventry, United Kingdom. *Correspondence e-mail: mvbdias@usp.br

Adenylate-forming enzymes (AFEs) are a mechanistic superfamily of proteins that are involved in many cellular roles. In the biosynthesis of benzoxazole antibiotics, an AFE has been reported to play a key role in the condensation of cyclic molecules. In the biosynthetic gene cluster for the benzoxazole AJI9561, AjiA1 catalyzes the condensation of two 3-hydroxyanthranilic acid (3-HAA) molecules using ATP as a co-substrate. Here, the enzymatic activity of AjiA1 is reported together with a structural analysis of its apo form. The structure of AjiA1 was solved at $2.0 \AA$ resolution and shows a conserved fold with other AFE family members. AjiA1 exhibits activity in the presence of 3-HAA $\left(K_{\mathrm{m}}=77.86 \pm 28.36, k_{\mathrm{cat}}=0.04 \pm 0.004\right)$ and also with the alternative substrate 3 -hydroxybenzoic acid (3-HBA; $\left.K_{\mathrm{m}}=22.12 \pm 31.35, k_{\mathrm{cat}}=0.08 \pm 0.005\right)$. The structure of AjiA1 in the apo form also reveals crucial conformational changes that occur during the catalytic cycle of this enzyme which have not been described for any other AFE member. Consequently, the results shown here provide insights into this protein family and a new subgroup is proposed for enzymes that are involved in benzoxazole-ring formation.

\section{Introduction}

The adenylation reaction catalyzed by adenylate-forming enzymes (AFEs) is ubiquitous and plays crucial metabolic or catabolic roles in all organisms, including fatty-acid metabolism (Hisanaga et al., 2004), detoxification (Chang et al., 1997), bioluminescence (Conti et al., 1996; Day et al., 2009; Gulick, 2009) and in the biosynthesis of amino acids, proteins and natural products (Stanišić \& Kries, 2019; Kudo et al., 2019; Labby et al., 2015; Huang et al., 2012; Lv et al., 2015; Cusack, 1997). AFE members are classified into three major classes, in which those from class I or the AFN family are the most diverse and include NRPS (nonribosomal peptide synthase) adenylation domains (subclass Ia), acyl- or aryl-CoA synthetases (subclass $\mathrm{Ib}$ ), and oxidoreductases and firefly luciferases (subclass Ic). However, several members of the class I AFEs, including enzymes involved in the degradation of organic molecules, such as PaaK1 and PaaK2 from Burkholderia cenocepacia (Law \& Boulanger, 2011) and 2-hydroxyisobutyric acid-CoA ligase (HCL) from Aquincola tertiaricarbonis (Zahn et al., 2019), are not suitable for this classification and they are classified into an alternative subgroup of class I AFEs.

Although class I AFE members show divergence in structural features and function, they all catalyze the same general 
biochemical transformation (Lux et al., 2019; D'Ambrosio \& Derbyshire, 2020). AFEs catalyze this reaction in two steps: they first catalyze the ATP-dependent activation of a carboxylic acid substrate as a reactive acyl adenylate (acylAMP) intermediate, which is followed by the binding of the enzyme by an acceptor molecule and the consecutive transfer of the acyl adenylate to a nucleophilic $\mathrm{O}, \mathrm{S}$ or $\mathrm{N}$ atom, generating ester, thioester and amide products, respectively (Lux et al., 2019; D'Ambrosio \& Derbyshire, 2020; Duckworth et al., 2012).

Structurally, the active site is at the interface of N-terminal and C-terminal domains, which are connected by a flexible hinge (Schmelz \& Naismith, 2009; D'Ambrosio \& Derbyshire, 2020). To catalyze the adenylation reaction, the class I AFEs undergo extensive conformational changes during the second half of the reaction (Lux et al., 2019). Generally, these enzymes undergo a domain rotation to introduce opposing faces of the dynamic C-terminal domain for the active-site reactions (Gulick, 2009). Hence, two distinct conformations are observed in AFEs, in which the orientation of the C-terminal domain differs by about $140^{\circ}$. Consequently, the C-terminal domain is involved in the formation of two distinct active-site architectures, a phenomenon known as domain alternation (Reger et al., 2008; Gulick, 2009; Bandarian et al., 2002).
Although the class I AFEs play a crucial role in the biosynthesis of natural products, particularly nonribosomal peptides from NRPSs that require an adenylation domain during the incorporation and extension of the peptide chain, a number of NRPS-independent AFEs with modest sequence similarity to phenylacetate-CoA ligases such as PaK1 and HCL have recently been identified in the biosynthetic gene clusters (BGCs) for benzoxazole natural products, including nataxazole (Cano-Prieto et al., 2015), caboxamycin (Losada et al., 2017), A33853 (Lv et al., 2015), calcimycin (Wu et al., 2011) and AJI9561 (gene cluster not published). In particular, AJI9561, which was isolated from Streptomyces sp. AJ9561, is structurally similar to UK-1 and nataxazole and contains a backbone composed of two molecules of 3-hydroxyanthranilic acid (3-HAA) and a 6-methylsalicylic acid (6-MSA) unit (Sato et al., 2001; Cano-Prieto et al., 2015; Fig. 1).

The BGCs for oxazole-containing natural products indicate the essential participation of a series of unusual enzymes, including an ATP-dependent coenzyme A ligase and an amidohydrolase, and their biosynthetic roles in the formation of this structurally unique class of natural products have remained unexplored until recently (Lv et al., 2015).

In the biosynthesis of A33853, the ATP-dependent coenzyme A ligase BomJ catalyzes the adenylation of 3-HAA and the subsequent production of a 3-HAA dimer. This is the<smiles>Cc1cccc(O)c1-c1nc2c(-c3nc4c(C(=O)O)cccc4o3)cccc2o1</smiles>

AJI9561

(a)

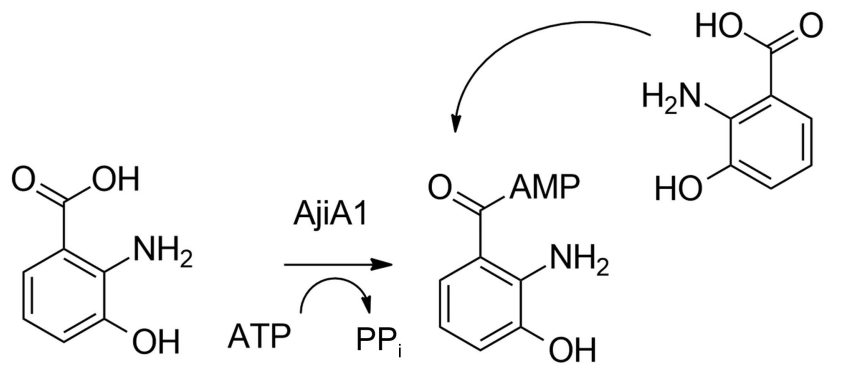<smiles>Nc1c(O)cccc1C(=O)Nc1c(O)cccc1C(=O)O</smiles>

3-Hydroxyanthranilic acid (3-HA)

3-HA dimer

3-HA-oxazole dimer

Proposed function of proteins

AjiA1: ATP-dependent ligase

AjiH: (ATP-dependent) amidohydrolase

Figure 1

Chemical structure of AJI9561 and the proposed reaction catalyzed by AjiA1 and the hypothetical amidohydrolase AjiH. (a) The structure of AJI9561, which contains two benzoxazole rings and a unit derived from 6-MSA. (b) AjiA1 conducts an ATP-dependent activation and the dimerization of 3-HAA, wihile the hypothetical aminohydrolase $\mathrm{AjiH}$ performs the closure of the oxazole ring, producing the benzoxazole ring. 
substrate for the next step in the pathway, which is catalyzed by the amidohydrolase BomN that assembles the benzoxazole ring through closure of the oxazole moiety. In the BGC for the biosynthesis of AJI9561, an AFE member, here named AjiA1, was identified and plays a similar role to BomJ in the formation of a 3-HAA dimer; a further hypothetical amidohydrolase should complete the assembly of the benzoxazole ring (Fig. 1a; Lv et al., 2015).

Here, we have investigated the structure of AjiA1 in its uncomplexed state and performed a structural analysis of this enzyme in comparison with the previously solved structures of its orthologs in complex with several ligands. Additionally, we have also used an in vitro approach to test the precursor 3-HAA and the alternative substrate 3-hydroxybenzoic acid (3-HBA).

\section{Methods}

2.1. Heterologous expression of AjiA1 in Escherichia coli and purification

The ajiA1 gene was cloned into pET-28a in frame with an $\mathrm{N}$-terminal polyhistidine tag and this construct (pET-28aajiA1) was used to transform chemically competent $E$. coli BL21 (DE3) cells (Thermo Fisher, USA). A single colony of the E. coli BL21 (DE3) transformant carrying the pET-28aajiA1 plasmid was cultured at $37^{\circ} \mathrm{C}$ and $200 \mathrm{rev} \mathrm{min}^{-1}$ in lysogeny broth supplemented with $50 \mu \mathrm{g} \mathrm{ml}^{-1}$ kanamycin. Once the culture reached an $A_{600}$ of 0.6 , protein expression was induced by adding isopropyl $\beta$-D-1-thiogalactopyranoside to a final concentration of $1 \mathrm{~m} M$. The culture was incubated

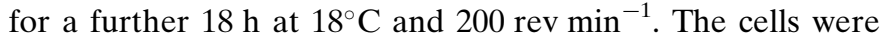
harvested by centrifugation ( $7000 \mathrm{~g}$ for $30 \mathrm{~min}$ at $4^{\circ} \mathrm{C}$ ) and resuspended in $30 \mathrm{ml} 50 \mathrm{~m} M$ HEPES $\mathrm{pH} 8,300 \mathrm{~m} M \mathrm{NaCl}$, $10 \%$ glycerol. The cells were lysed using an ultrasonic sonicator (Branson Sonifier) at $30 \%$ amplitude, with a pulse every $1 \mathrm{~s}$ for $6 \mathrm{~min}$. The insoluble fraction was removed by centrifugation $\left(21700 \mathrm{~g}\right.$ for $60 \mathrm{~min}$ at $\left.4{ }^{\circ} \mathrm{C}\right)$ and the soluble fraction was passed through a HisTrap HP $5 \mathrm{ml}$ nickel-affinity column (GE Healthcare) to bind the recombinant protein. Lysis buffer containing increasing imidazole concentrations $(25-500 \mathrm{mM})$ was used to remove contaminants and subsequently to elute the pure recombinant protein (monitored by SDS-PAGE). Fractions containing AjiA1 were concentrated using an Amicon Ultra centrifugal filter unit (Merck Millipore, USA). The protein concentration was determined using a BioDrop $\mu$ Lite (Biochrom). The protein was further purified using a preparative gel-filtration HiLoad 16/60 column packed with Superdex 75 resin (GE Healthcare Lifescience, USA). For this purification, buffer consisting of $100 \mathrm{~m} M$ bis-Tris propane $\mathrm{pH}$ $6.5,150 \mathrm{mM} \mathrm{KCl}$ was used. Protein fractions were pooled, concentrated, flash-frozen in liquid nitrogen and stored at $-80^{\circ} \mathrm{C}$.

\subsection{Protein crystallization}

AjiA1 at $8 \mathrm{mg} \mathrm{ml}^{-1}$ was crystallized using the hanging-drop vapour-diffusion method at $18^{\circ} \mathrm{C}$ in a condition composed of
$100 \mathrm{~m} M$ potassium phosphate citrate $\mathrm{pH} 4.2$ buffer, $35 \%$ PEG 300. Crystals were harvested from the crystallization drop and transferred into cryoprotectant solution consisting of the crystallization solution and $15 \%$ ethylene glycol. The crystals were then flash-cooled in liquid nitrogen using nylon loops. $X$-ray diffraction data were collected on beamline 13 at PETRA III, DESY, Hamburg, Germany. The X-ray data were processed using XDS (Kabsch, 2010) and scaled using AIMLESS (Evans \& Murshudov, 2013) from the CCP4 suite (Winn et al., 2011). The phase was determined by molecular replacement using the structure of PaaK1 (PDB entry 2y27; Law \& Boulanger, 2011) as the search model with Phaser (McCoy, 2007) from the Phenix suite (Liebschner et al., 2019). The AjiA1 model was refined using phenix.refine (Afonine et al., 2012). Further visual inspection and real-space refinement were performed in Coot (Emsley et al., 2010). The stereochemical quality of the model was assessed using MolProbity (Chen et al., 2010). Figures showing protein structure were prepared using PyMOL (version 2.0; Schrödinger).

\subsection{Enzymatic assays}

Enzymatic assays were performed by a coupled continuous spectrophotometric assay using the EnzChek Pyrophosphate Assay Kit (Molecular Probes). The initial reaction mixtures consisted of $0.2 \mathrm{mM}$ 2-amino-6-mercapto-7-methylpurine ribonucleoside (MESG), $1 \mathrm{U}$ purine nucleoside phosphorylase, $0.01 \mathrm{U}$ inorganic pyrophosphatase, $10 \mathrm{~m} M$ ATP, $20 \times$ reaction buffer and AjiA1 $(8 \mu M)$ in a total volume of $100 \mu$ l. The reaction mixtures were pre-incubated at $25^{\circ} \mathrm{C}$ for $10 \mathrm{~min}$ and were initiated by addition of the substrate at various concentrations. The increase in the absorbance at $360 \mathrm{~nm}$ resulting from the phosphorolytic release of the purine base (2-amino-6-mercapto-7-methylpurine) from MESG was monitored over a $15 \mathrm{~min}$ period. The kinetic parameters $K_{\mathrm{m}}$ and $V_{\max }$ were calculated by fitting the initial rates to a nonlinear regression hyperbolic model under the assumption of a Michaelis-Menten kinetic model using the GraphPad Prism 5.01 software. For all measurements, three independent experiments were performed, each in triplicate.

\subsection{Alignment analysis and phylogeny}

The AjiA1 protein sequence was aligned against those of 12 AFE family members that have been structurally characterized (sequences were obtained from the Protein Data Bank; https://www.rcsb.org/; Berman et al., 2000). Alignments were performed using Clustal Omega (Sievers et al., 2011) and were imported into Jalview version 2.11.0 (Waterhouse et al., 2009). The members of this enzyme family were chosen based on the conserved sequence motifs in the ANL superfamily of enzymes (Marahiel et al., 1997; Gulick, 2009). The evolutionary history was inferred using the maximum-likelihood method and the JTT matrix-based model (Jones et al., 1992). The tree with the highest log-likelihood $(-17500.85)$ was chosen. Initial tree(s) for the heuristic search were obtained automatically by applying the neighbour-joining and BIONJ algorithms to a matrix of pairwise distances estimated using a 
JTT model and then selecting the topology with the superior log-likelihood value. Finally, a phylogenetic tree was constructed using MEGA X (Kumar et al., 2018).

\section{Results and discussion}

\subsection{Overall structure of AjiA1}

The structure of AjiA1 was solved at a resolution of $2.0 \AA$. Attempts to obtain crystals of the protein in complex with ATP were unsuccessful, and co-crystallization with substrate or substrate analogs did not show the corresponding electron density in the active site. The structure was solved by mole-

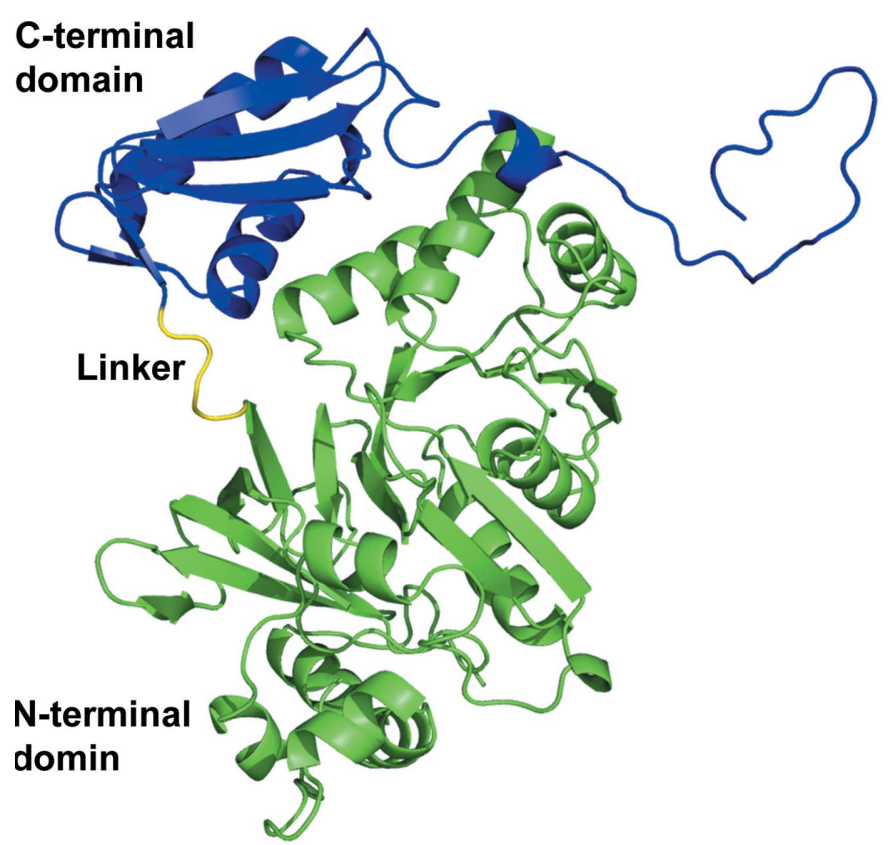

$(a)$

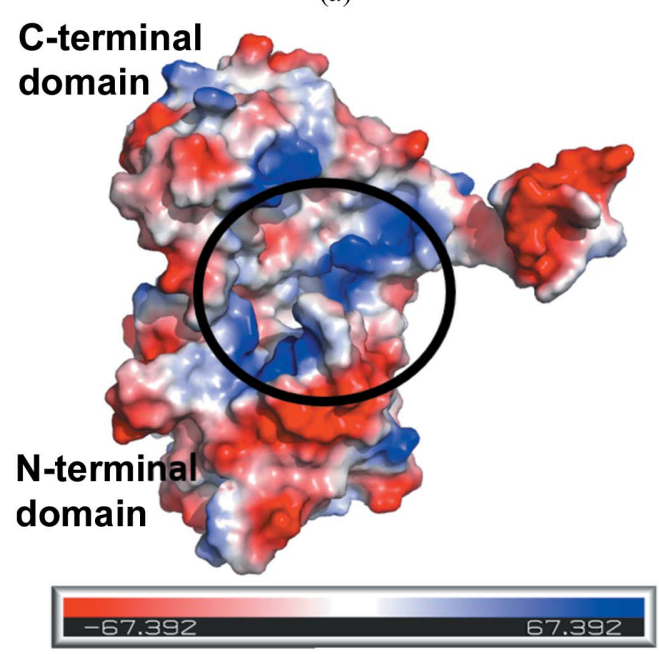

(b) cular replacement using the structure of PaaK1 (PDB entry 2y27; Law \& Boulanger, 2011) as a search model. The AjiA1 crystals belonged to space group $P 3_{1} 21$, with two protomers in the asymmetric unit (r.m.s.d. of $1.21 \AA$ ), which should correspond to the functional homodimeric quaternary structure of the protein in solution as predicted by PISA (Krissinel \& Henrick, 2007; Fig. 2). Further data-processing and crystallographic statistics are shown in Supplementary Table S1.

Similar to other members of the AFE family, AjiA1 has an overall architecture constituted of two $\alpha / \beta$ domains with a central core of parallel $\beta$-sheets surrounded by an outer layer of $\alpha$-helices, with the active site formed at the interface of the $\mathrm{N}$ - and C-terminal domains (Fig. 2c; Branden \& Tooze, 2012). AjiA1 lacks the typical $\alpha / \beta$-sandwich arrangement at the $\mathrm{N}$-terminus which is common to other AFE family members, and has a small helical bundle microdomain (Supplementary Fig. S1; Law \& Boulanger, 2011). Each AjiA1 protomer, as expected, consists of a large $\mathrm{N}$-terminal domain ( $\mathrm{N}$ domain; Ser4-Gly321) and a small C-terminal domain (C domain;
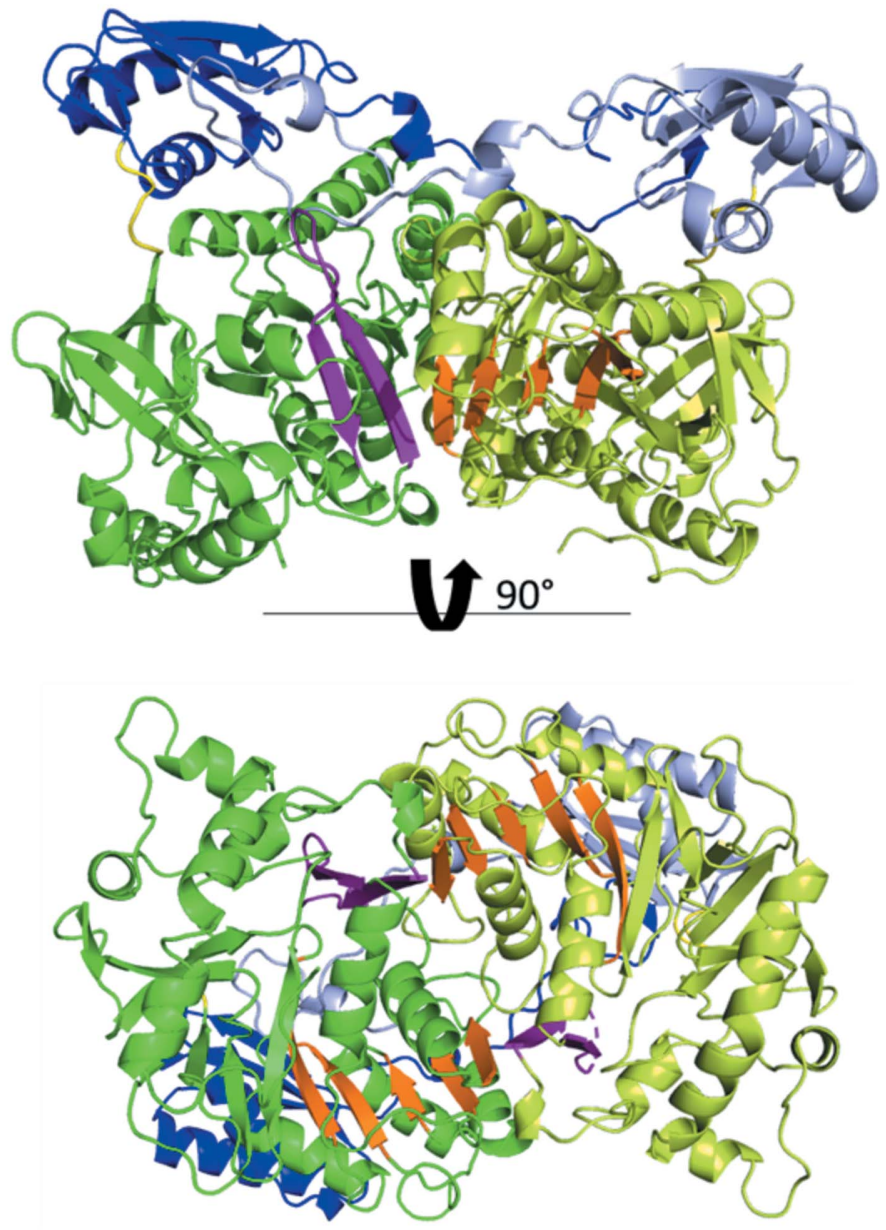

(c)

Figure 2

The overall structure of AjiA1. (a) The structure of the AjiA1 monomer with the larger $\mathrm{N}$ domain in green, the C domain in blue and the linker that connects the domains in yellow. (b) The electrostatic potential surface of the AjiA1 monomer. Blue indicates positive charge and red indicates negative charge. The black circle indicates the active-site groove. (c) Dimerization of AjiA1. Intimate dimerization interactions of AjiA1 create composite $\beta$-sheets spanning both monomers. Two strands of the first protomer (purple) are abutted by the distorted five strands of the second protomer, which are represented in orange. The larger $\mathrm{N}$ domain is represented in green for the first protomer and in lime for the second protomer. The $\mathrm{C}$ domain of the first protomer is shown in blue and that of the second protomer is shown in light blue; the linker that connects the two domains is shown in yellow. 
His327-Ala432) (Fig. 2a). The N domain of AjiA1 consists of three antiparallel two-stranded $\beta$-sheets sandwiched by nine $\alpha$-helices, whereas the $\mathrm{C}$ domain is defined by two helices, a short antiparallel two-stranded $\beta$-sheet, a twisted threestranded sheet and a long loop that extends to the adjacent protomer. Analysis of the electrostatic potential surface of the dimer indicates a positively charged cavity at the interface of the $\mathrm{N}$ and $\mathrm{C}$ domains, as observed previously for other AFE family members (Marahiel et al., 1997; Law \& Boulanger, 2011; Bera et al., 2010; Nakatsu et al., 2006; Fan et al., 2016; Shao et al., 2019; Fig. 2b).

AjiA1 forms a dimer with two-axis symmetry, which is stabilized by the formation of two large seven-stranded intersubunit $\beta$-sheets with contributions from two antiparallel strands from one protomer and five parallel strands from the second protomer (Fig. 2c). The dimer interface is about $\sim 3999 \AA^{2}$ and is stabilized through extensive shape and chemical complementarity (Supplementary Fig. S2), including extensive hydrophobic interactions, by 46 hydrogen bonds and eight salt bridges (Supplementary Fig. S3). However, similarly to other AFE family members, most of the $\mathrm{C}$ domain is not involved in dimerization, apart from the long C-terminal loop that extends to the adjacent protomer (Fig. 2c).

AjiA1 shares $30 \%$ sequence identity and $91 \%$ sequence coverage with PaaK1, a phenylacetate-CoA ligase involved in the biosynthesis of phenylacetic acid (Law \& Boulanger, 2011; Holm \& Laakso, 2016). Backbone superposition of AjiA1 and PaaK1 gave an r.m.s.d. of $2.5 \AA$, indicating that the structures are reasonably different (Fig. 3). Recently, the structure of the ATP-dependent ligase NatL2 involved in the biosynthesis of nataxazole was also solved but in complex with ATP and substrate; it shares a sequence identity of about $91 \%$ with AjiA1 (Song et al., 2020). Superposition of these two structures gave an r.m.s.d. of about $1.14 \AA$, indicating high structural similarity (Fig. 3). The large $\mathrm{N}$ domain overlaps reasonably well with those of AjiA1 and other AFEs, while

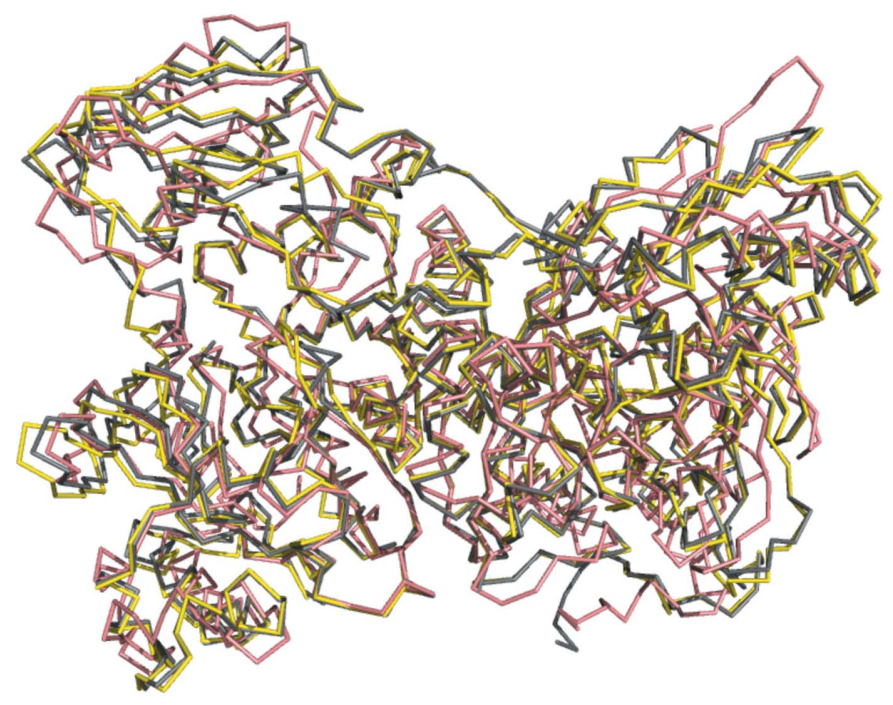

Figure 3

Superposition of AjiA1 (yellow) with its closest homologs PaaK1 (salmon) and NatL2 (grey). other regions show significant differences, particularly in the active-site region, which is located in a groove between the $\mathrm{C}$ and $\mathrm{N}$ domains (Supplementary Fig. S4). All of the conserved signature motifs (A1-A10) that are commonly observed in adenylation domains (Marahiel et al., 1997) are conserved in AjiA1, including the P-loop, the linker motif (A8) and the adenine motif (A5), which contains critical residues for binding the adenine moiety of ATP/AMP (Supplementary Fig. S5).

\subsection{Binding of metal ions}

During structure refinement, we observed a zinc ion coordinated by Cys248, Cys308, Cys310 and His 254 in AjiA1 (Supplementary Fig. S6). As this ion is located remotely from the active site, it should only play a structural role, probably in reducing side-chain movement and conformational changes, as has also been proposed in the structural analysis of NatL2 (Song et al., 2020). Compared with other homologs, the $\mathrm{Zn}^{2+}$ ion is only present in AjiA1 and NatL2 and consequently should be a particular characteristic of this group of AFEs.

Furthermore, several of the previously reported structures of AFE family members contain an $\mathrm{Mg}^{2+}$ ion coordinated in the active site, acting directly in ATP catalysis (Reger et al., 2008). AjiA1 has an $\mathrm{Mg}^{2+}$-binding site in each chain, although again remote from the active site, which should also play a structural role in reducing the structural flexibility of the region (Supplementary Fig. S7). Structural analysis of AjiA1 did not indicate the presence of any metal in the active site, in contrast to the other AFE structures in complex with ATP or ATP analogs, and consequently this ion should be associated with the co-substrate.

3.3. Active-site architecture, C-terminal loop swapping and comparison with other AFE family members

Although structures of NatL2 have been solved in complex with AMPPNP and with AMP in the presence of the substrate 3-HAA, the structure of the apo form has not been reported. In addition, as the structures of AFE family members undergo different motions on co-substrate and substrate binding, analysis of the conformational changes during the enzymatic catalytic cycle, including the unbound state, is necessary in order to completely understand the dynamics of these enzymes in different complexes.

The active site of AjiA1 is located in a groove at the interface of the $\mathrm{N}$ and $\mathrm{C}$ domains. In contrast to most AFE structures solved to date, residues from both protomers of the AjiA1 dimer are involved in catalytic site formation and the dimeric structure should be essential for enzyme activity, which is a novel feature in this class of enzymes. Although the key catalytic residue Lys 418 , which should play a role in nucleophilic attack on the $\alpha$-phosphate of ATP, superposes well with the catalytic Lys422 in PaaK1, in AjiA1 it comes from the adjacent protomer through loop swapping. The observed loop swapping might reflect not only the dynamics of the protomers but also a peculiarity of AFEs involved in the formation of benzoxazole rings, as a similar event has also 
been observed in NatL2 (Schiering et al., 2000; Song et al., 2020; Fig. 4).

Generally, loop swapping is associated with an unconventional mechanism of oligomerization in which the structural elements of individual protomers are interchanged between identical partners by recruiting interactions that are crucial for stabilizing the protein. The phenomenon has been proposed to play a role in aggregation, misfolding and allosteric regulation, but its biological implications remain unclear (Yang et al., 2004). Initially, we would expect that the co-substrate binding triggered movements in this region that are needed for catalytic activity. However, the apo structure of AjiA1 indicates that this loop does not return to its protomer in the absence of ligand, suggesting that this structural feature is not responsible for allosteric regulation. In contrast, it should play a role in stabilization of the dimer or in decreasing the flexibility of the $\mathrm{C}$ domain, in contrast to the $\mathrm{C}$ domains of other AFE family members, including PaaK1 (Law \& Boulanger, 2011) and HCL (Zahn et al., 2019), which are reported to undergo drastic conformational changes. Nevertheless, amino-acid residues Leu422-Ala424 of chain $A$ form a two-stranded parallel $\beta$-sheet with amino acids Ala353-Ala359 of chain $B$, and vice versa, in the loop-swapping region (Supplementary Fig. S8).

The ATP-binding site contains several highly conserved motifs that are present in all family members and perform similar roles in positioning the nucleotide. Hence, the key catalytic residues, including Arg322 from the A8 motif, which is proposed to be involved in stabilization of the reaction intermediate and is located in a short and solvent-exposed interdomain linker (Gulick, 2009), Asp299 from the A7 motif, which is universally conserved and interacts with both ribose hydroxyls, and Glu240, a residue from the A5 motif, should be involved in anchoring the adenylate intermediate. An aromatic residue from the A5 motif, Tyr236, stacks against the adenine base.

The major differences in the active site that are observed on the superposition of AjiA1 and NatL2-AMPPNP are the proximity of Ser88 (from the P-loop) and Lys418 (the A10 lysine) to the AMP phosphate group and a repositioning of Ala212 close to the adenine ring, as well as the participation of six water molecules (Fig. 5a).

Further structural analysis shows, as expected, that several functional regions of this protein also have highly conserved

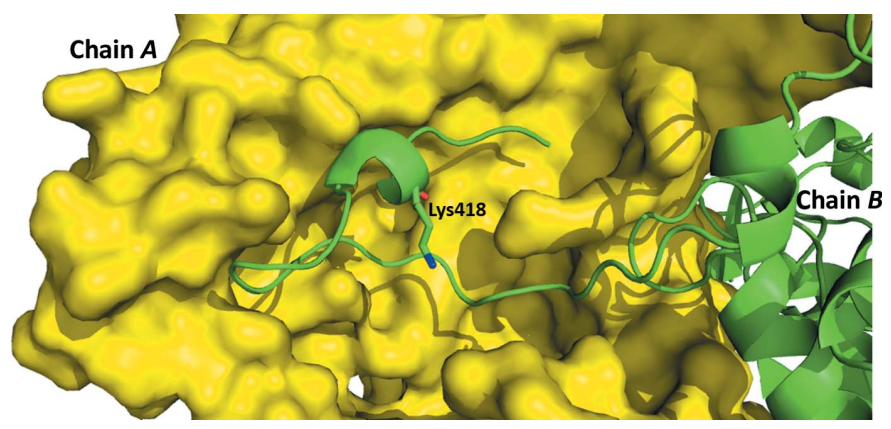

Figure 4

Loop swapping inAjiA1. The conserved residue Lys418 is shown in stick representation. conformations (Supplementary Fig. S9). Other amino-acid residues which are in the active-site pocket region, including motifs A3 or the P-loop, A5, A6 and A7, are also substantially conserved (Ashkenazy et al., 2016; Marahiel et al., 1997). However, based on the conserved active-site residues, AjiA1 has similar catalytic residues to those reported for PaaK1, HCL and NatL2. Consequently, AjiA1 should have a similar catalytic mechanism to that observed in other AFEs, which involves adenylation of the carboxylic acid of the substrate followed by an esterification reaction with a second molecule of substrate, which comprises the ring condensation of the AJI956 intermediate.

However, AjiA1 also has several differences when compared with other AFE members. Lys418 and Tyr435 in the C-terminal loop swapping are more buried and are closer to the 3-HAA molecules in the active site of the NatL2 complex, and they have considerable conformational changes when compared with the same residues in the structure of AjiA1. Arg156 also undergoes a relevant conformational change as it moves away from the active site in AjiA1, while Glu235 from motif A5 seems to play a role in positioning 3-HAA in the active site, contributing to a hydrogen bond to the substrate hydroxyl group. In the complex of NatL2 with 3-HAA, Thr135 also undergoes a rotation in comparison to AjiA1 and its side chain makes a hydrogen bond to one of the 3-HAA molecules (Fig. $5 b$ ).

A comparison of the structures of AjiA1, NatL2-AMPPNP (r.m.s.d. of $1.12 \AA$ ) and NatL2-AMP-3-HAA (r.m.s.d. of $1.14 \AA$ ) revealed key motions that should occur during the catalytic cycle and also owing to the binding of co-substrate. A series of conformational changes in several loops of the active site are observed when the NatL2-AMP complex and the uncomplexed state of AjiA1 are superposed. The largest changes are observed in six loops near the active site: Glu86Pro94 (motif A3, P-loop), Thr210-Glu213, Ser238-Gly242 (motif A5), Asn296-Ala298 (motif A7), Thr305-Leu313 and Leu320-Thr328 (motif A8) in AjiA1 (Fig. 5c). Three of these five loops have higher $B$ factors (Glu86-Pro94, Thr210Glu213 and Leu320-The328) in comparison to other regions of NatL2 and consequently should reflect the highest flexibility in AjiA1. Thus, the average displacement suggests that the most flexible part of the protein should act in the catalytic function. In contrast, the core fold in the $\mathrm{N}$ domain is highly conserved and rigid, as shown by its low average $B$ factor (Supplementary Fig. S10).

The P-loop (motif 3) of AjiA1, which includes Ser87-Pro94 (Supplementary Fig. S5), is a highly conserved region in all AFE family members and has the amino-acid sequence $\mathrm{G} X_{4} \mathrm{GK}(\mathrm{TS})$. This region is proposed to play a bifunctional role in the structure of PaaK1 involving both coordination of ATP and stabilization of the C domain. In the PaaK1 structure in complex with an adenylate intermediate without the pyrophosphate group, this region adopts an opened and disordered structure. In contrast, on binding the co-substrate ATP this region adopts a closed and ordered conformation, establishing hydrogen-bond contacts with the $\beta$ - and $\gamma$-phosphates of the co-substrate. Although NatL2 has been crystallized in the 
presence of AMP, the position of the P-loop differs considerably from that in AjiA1. In AjiA1 the P-loop adopts an opener conformation with a displacement of about $3 \AA$ in comparison to NatLA2 and moves towards the C-terminal loop. Additionally, the $\mathrm{N}$ domain also seems to adopt a closer conformation to the active site by about $1.3 \AA$ (Fig. $5 c$ ). The $\mathrm{C}$ domain, and consequently the C-terminal loop, also moves to a position closer to the active site by about $1.5 \AA$, allowing Lys418 to adopt its catalytic position and allowing it to interact with the phosphate and ribose moieties of AMP (Fig. 5d).

In the reaction catalyzed by the enzymes within this family, a substrate carboxylate is activated using ATP to form an acyladenylate (Reger et al., 2008). Structural and functional studies indicated that these enzymes alternate between two conformations during catalysis (Reger et al., 2008; Shao et al., 2019; Nakatsu et al., 2006; Gulick, 2009). The presence of the catalytic lysine in the loop swapping would suggest an intense movement of this 'arm', which functions as a 'gate' for the open and closed structures; however, our apo structure did not show evidence of a large conformational change in this region and the $\mathrm{C}$ domain does not adopt the closed and open conformations described for other members of AFE family. In contrast, the long C-terminal loop should play a role in locking the structure in its closed state as observed by Song et al. (2020). Interestingly, as loop swapping plays a role in locking the structures of AjiA1 and NatL2 in the fixed and closed state, large conformational motions in the $\mathrm{C}$ domain are unlikely and hence domain alternation should not occur. Since large conformational changes involve a higher enthalpic energetic barrier, these enzymes could have evolved differently and adopt an elegant strategy to perform the two halfreactions commonly observed in AFEs.

The loop swapping observed in these structures could maintain both half-reactions in a single active site, avoiding

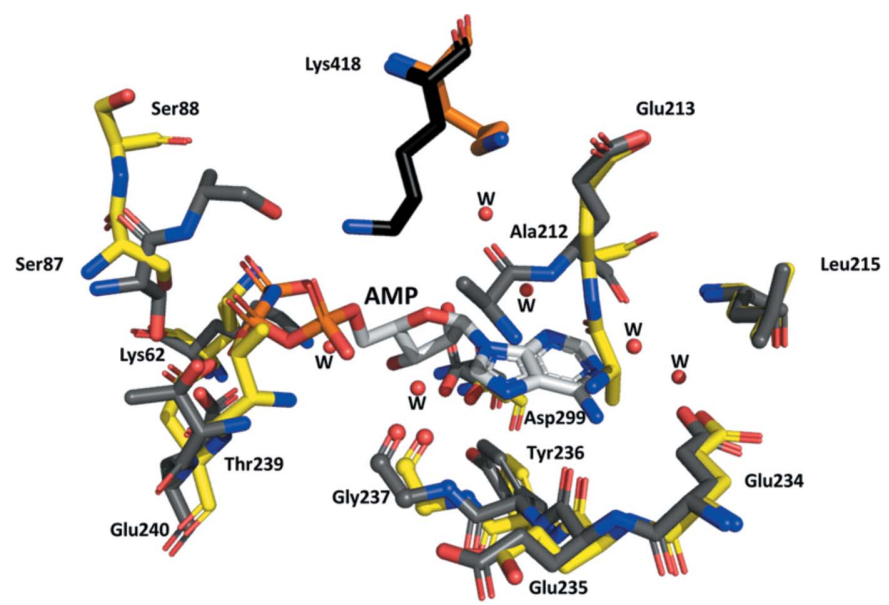

(a)

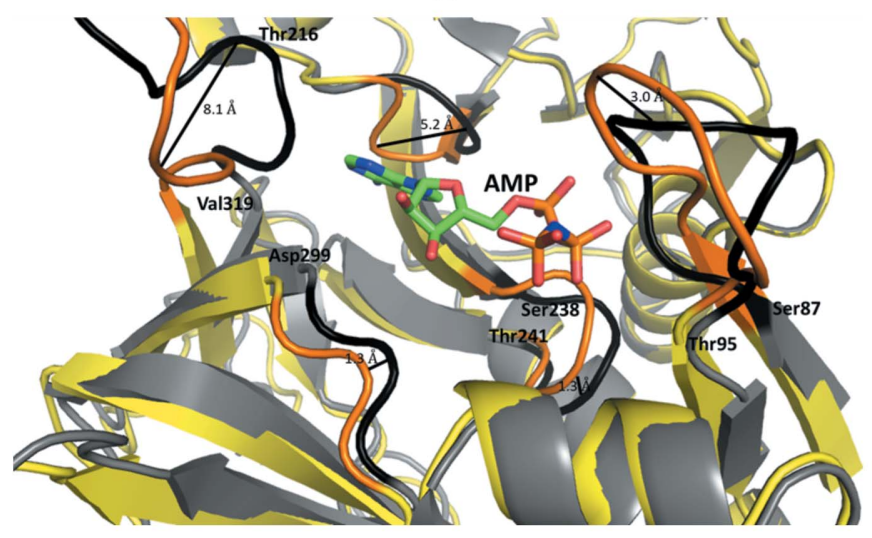

(c)

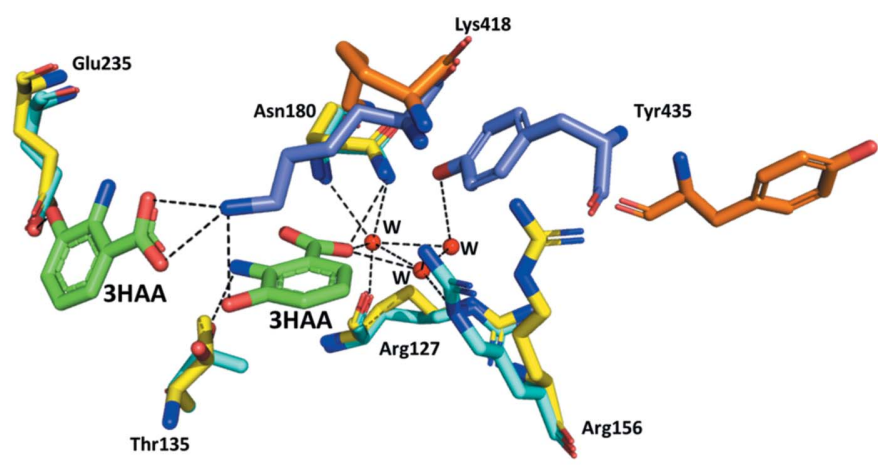

(b)

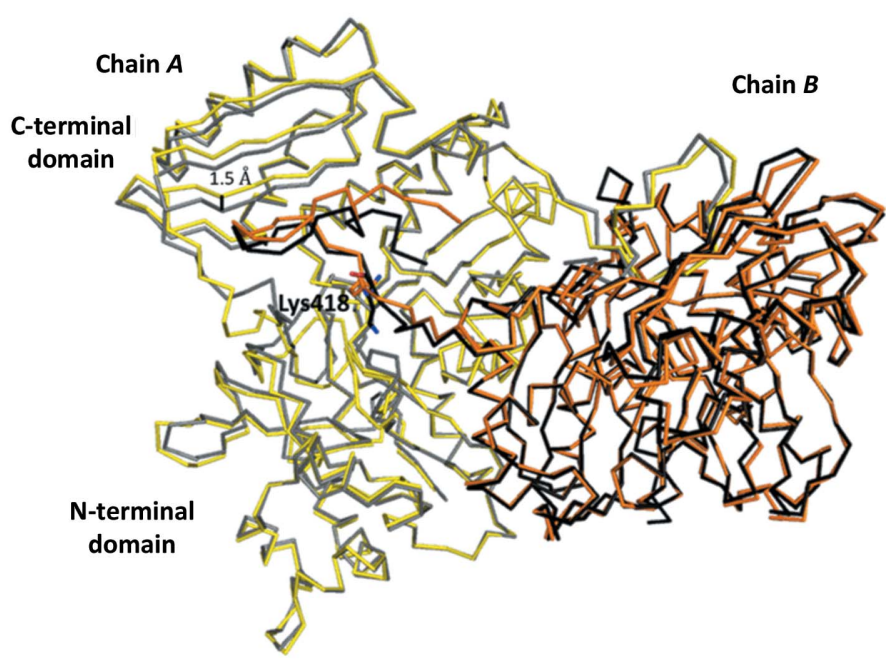

(d)

Figure 5

Comparison of AjiA1 with structures in complex with AMP and substrates. (a) Superposition of AjiA1 and NatL2-AMPPNP (PDB entry 6six). The AjiA1 residues are represented in yellow and those from NatL2 are in grey. The key catalytic residue Lys418 is in a stronger colour as it belongs to the adjacent protomer. (b) Superposition of AjiA1 and NatL2-AMP-3-HAA (PDB entry 6siy). AjiA1 residues are represented in yellow, with Lys418 and Tyr435 in orange, and NatL2 residues are in cyan, with Lys418 and Tyr435 in blue. The different colours of these amino-acid residues indicate that they are from the adjacent protomer. Active-site waters are represented by red spheres. (c) Superimposition of AjiA1 and NatL2 in complex with AMPPNP (PDB entry 6six). The protomers are shown as a cartoon. AjiA1 is in yellow and its five different loops are shown in orange. NatL2-AMPPNP is in grey and its loops are in black. $(d)$ Superimposition of AjiA1 and NatL2 in complex with AMPPNP (PDB entry 6six). The protomers are represented as ribbons. AjiA1 is represented in yellow (chain $A$ ) and orange (chain $B$ ) and NatL2 in complex with AMPPNP (PDB entry 6six) is represented in grey (chain $A$ ) and black (chain $B$ ). The key catalytic residue Lys418 from AjiA1 and NatL2-AMPPNP is shown in stick representation. The C domain, and consequently the C-terminal loop, also moves to a position closer to the active site by about $1.5 \AA$. 


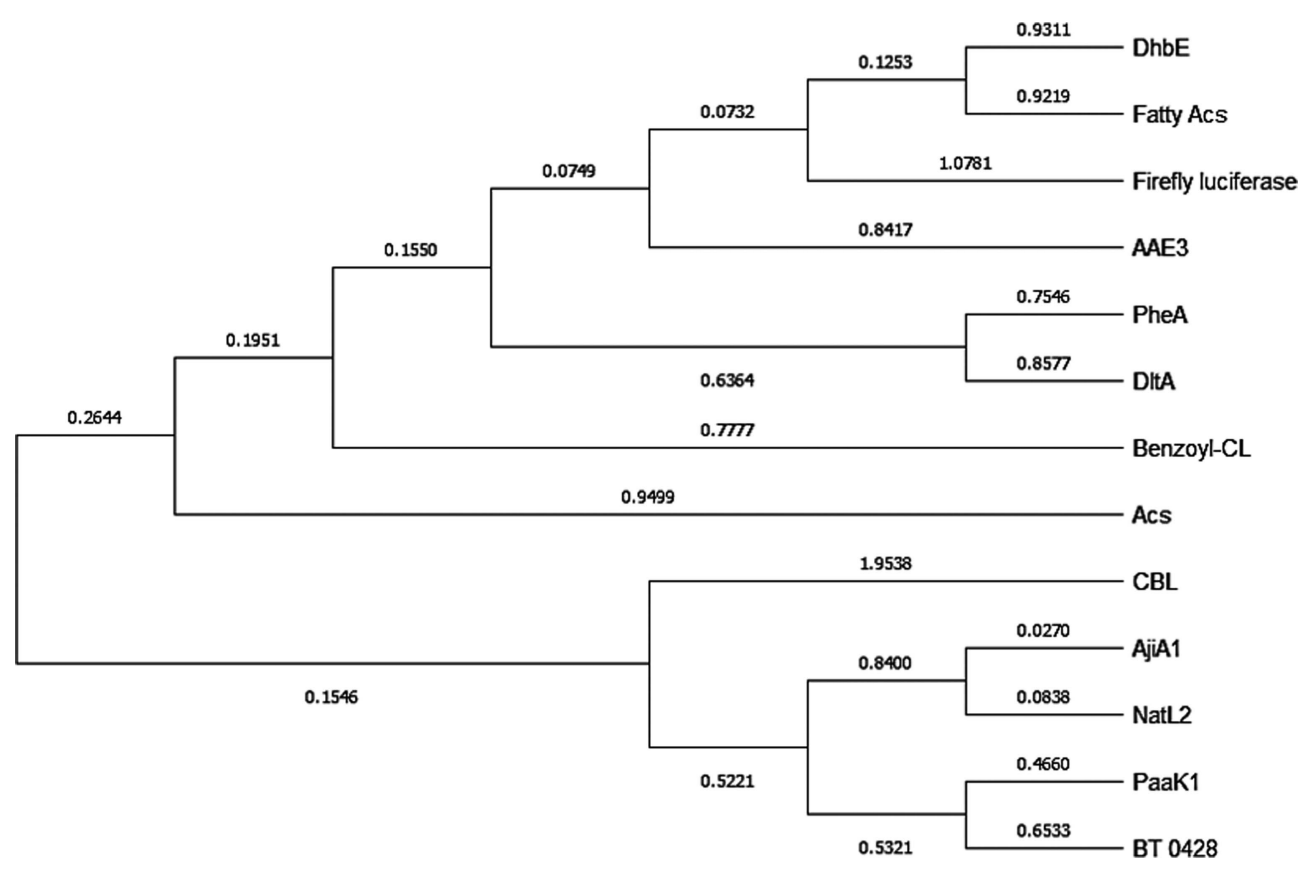

Figure 6

Evolutionary analysis by the maximum-likelihood method of several AFE family members that have been structurally characterized, including AjiA1. The branch lengths are shown. The proteins and organisms used are DhbE from Bacillus subtilis (PDB entry 1mdf), fatty acyl-CoA synthetase (fatty Acs) from Thermus thermophilus (PDB entry 1ult), firefly luciferase from Photinus pyralis (PDB entry 1lci), AAE3 from Arabidopsis thaliana (PDB entry 5ie3), PheA from Brevibacillus brevis (PDB entry 1amu), DltA from Bacillus cereus ATCC 14579 (PDB entry 3dhv), benzoyl-CoA ligase (benzoyl-CL) from Paraburkholderia xenovorans LB400 (PDB entry 2v7b), acetyl-CoA synthetase (Acs) from Salmonella enterica (PDB entry 1pg4), 4-chlorobenzoyl-CoA ligase/synthetase (CBL) from Alcaligenes sp. AL3007 (PDB entry 1t5h), AjiA1 from Streptomyces sp. AJ9561 (PDB entry 6wuq), NatL2 from Streptomyces sp. Tu 6176 (PDB entry 6six), PaaK1 from Burkholderia cenocepacia (PDB entry 2y27) and BT_0428 from Bacteroides thetaiotaomicron (PDB entry $4 \mathrm{r} 1 \mathrm{~m}$ ). The analyses were conducted in MEGA X (Kumar et al., 2018).
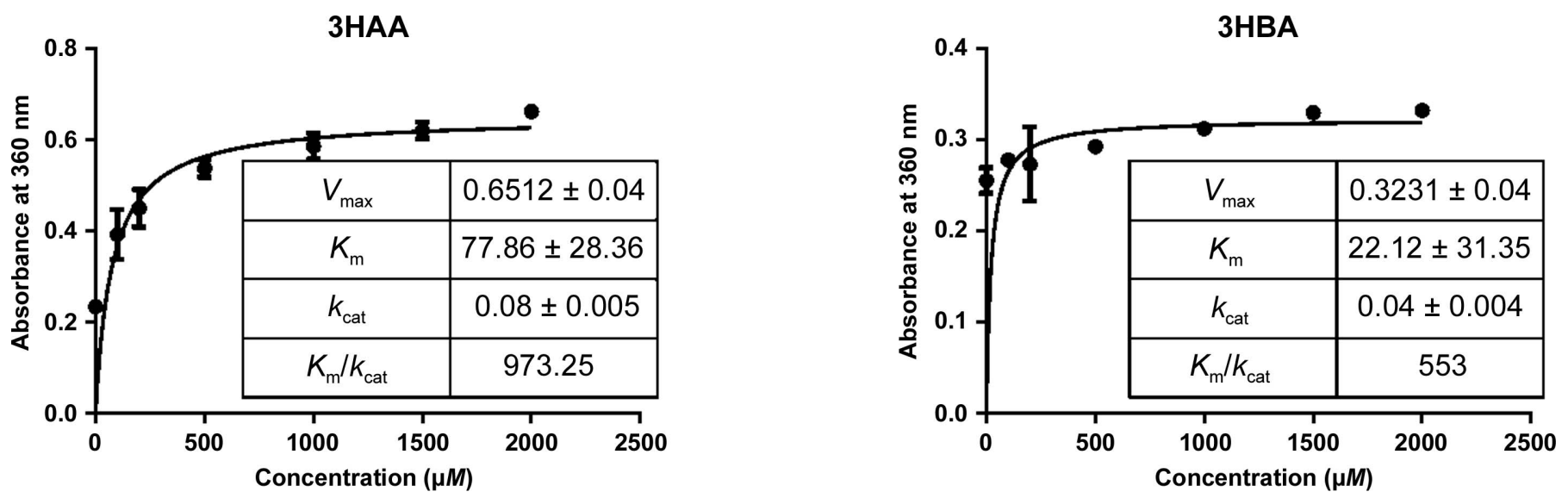

Figure 7

Kinetic analyses of AjiA1 were performed using 3-HAA and 3-HBA concentrations from 0 to $2500 \mu M$. $K_{\mathrm{m}}$ and $V_{\max }$ were determined from nonlinear regression to the Michaelis-Menten equation from three replicate experiments.

large rotations at the cost of a crucial dimeric quaternary structure. However, further investigation is needed to prove this hypothesis or to understand whether loop swapping plays an alternative role to the domain alternation observed in other class I AFE members.

\subsection{Phylogenetic analysis}

To gain insights concerning divergence and evolution, we performed a phylogenetic analysis using the maximum- likelihood method (Ziemert \& Jensen, 2012; Benevides et al., 2016), which reveals that AjiA1 and NatL2 can be grouped distinctly in a different branch (Fig. 6), supporting the postulation that these enzymes may have evolved independently and hence should be classified as a new subgroup of phenylacetate-CoA-like ligases.

\subsection{Kinectics and activity of AjiA1}

In order to gain insight into the promiscuity of AjiA1, which has a much more restrained structure, we performed an assay 
using the alternative substrate 3-hydroxybenzoic acid (3-HBA) and the natural substrate 3-HAA with ATP as a cosubstrate. We determined the kinetic parameters for 3-HAA and 3-HBA by measuring the increase in the absorbance at $360 \mathrm{~nm}$ using a spectrophotometric assay. As shown, the $K_{\mathrm{m}}$ and the catalytic efficiency $\left(k_{\text {cat }}\right)$ as well as the relationship $K_{\mathrm{m}} / k_{\text {cat }}$ decreased by approximately half when 3 -HBA was used as a substrate instead of 3-HAA. This indicates that the enzyme has a higher affinity but a lower catalytic efficiency for 3-HBA than for 3-HAA, the natural substrate. This result demonstrates that AjiA1 is able to perform at least the first part of the reaction and possibly to catalyze the formation of an ester bond in the absence of the amine group.

However, the results do not necessarily imply that an ester instead of an amide is formed when the native substrate 3-HAA is used (Fig. 7).

\section{Conclusion}

In summary, we have determined the structure of AjiA1 in its uncomplexed form and compared it with that of the enzyme NatL2 in complex with various ligands, which indicates that this group of enzymes has a completely new set of motions during the catalytic cycle compared with other AFE members. Domain alternation should not occur in enzymes involved in benzoxazole biosynthesis. In contrast, these enzymes have a domain-swapping event involving a long C-terminal loop. This loop constrains the $\mathrm{C}$ domain and locks the enzyme into a single conformation. Taking together the new movements, which involve only the loops of the active site and a slight rotation of the adjacent protomer, and phylogenetic analysis, we propose that these enzymes should be classified as a distinct group of phenylacetate-CoA-like ligases. In addition, even with the constraints imposed by the absence of domain alternation, AjiA1 was still able to accept alternative substrates, indicating promiscuity of these enzymes. Consequently, these findings provide new insights into the AFE family and indicate the possibility of using these enzymes in biotechnological approaches.

\section{Related literature}

The following references are cited in the supporting information for this article: Laskowski \& Swindells (2011) and Laskowski et al. (2018).

\section{Acknowledgements}

The authors declare that there are no conflicts of interest. Author contributions are as follows. MVBD and PFL designed the research. FCRP performed the protein production, purification, crystallization, structure determination and analysis. $\mathrm{KC}$ performed cloning and protein-expression optimization. MS performed the DNA-sequence analysis and AJ9561 gene cluster identification in the Streptomyces sp. AJ9561 whole genome. FCRP, KC and AMS performed the protein kinetics and analysis. FCRP and MVBD wrote the manuscript with contributions from and the approval of all authors.

\section{Funding information}

The following funding is acknowledged: Fundação de Amparo à Pesquisa do Estado de São Paulo (grant Nos. 2018/00351-1 and 2016/06034-2); Conselho Nacional de Desenvolvimento Científico e Tecnológico (grant Nos. 308351/2013-4, 404769/ 2018-7 and 302848/2017-7; studentship No. 141090/2016-2).

\section{References}

Afonine, P. V., Grosse-Kunstleve, R. W., Echols, N., Headd, J. J., Moriarty, N. W., Mustyakimov, M., Terwilliger, T. C., Urzhumtsev, A., Zwart, P. H. \& Adams, P. D. (2012). Acta Cryst. D68, 352367.

Ashkenazy, H., Abadi, S., Martz, E., Chay, O., Mayrose, I., Pupko, T. \& Ben-Tal, N. (2016). Nucleic Acids Res. 44, W344-W350.

Bandarian, V., Pattridge, K. A., Lennon, B. W., Huddler, D. P., Matthews, R. G. \& Ludwig, M. L. (2002). Nat. Struct. Biol. 9, 5356.

Benevides, L., Carvalho, D. S., Andrade, R. F. S., Bomfim, G. C. \& Fernandes, F. M. C. (2016). Genet. Mol. Biol. 39, 665-673.

Bera, A. K., Atanasova, V., Gamage, S., Robinson, H. \& Parsons, J. F. (2010). Acta Cryst. D66, 664-672.

Berman, H. M., Westbrook, J., Feng, Z., Gilliland, G., Bhat, T. N., Weissig, H., Shindyalov, I. N. \& Bourne, P. E. (2000). Nucleic Acids Res. 28, 235-242.

Branden, C.-I. \& Tooze, J. (2012). Introduction to Protein Structure. Boca Raton: CRC Press.

Cano-Prieto, C., García-Salcedo, R., Sánchez-Hidalgo, M., Braña, A. F., Fiedler, H. P., Méndez, C., Salas, J. A. \& Olano, C. (2015). ChemBioChem, 16, 1461-1473.

Chang, K.-H., Xiang, H. \& Dunaway-Mariano, D. (1997). Biochemistry, 36, 15650-15659.

Chen, V. B., Arendall, W. B., Headd, J. J., Keedy, D. A., Immormino, R. M., Kapral, G. J., Murray, L. W., Richardson, J. S. \& Richardson, D. C. (2010). Acta Cryst. D66, 12-21.

Conti, E., Franks, N. P. \& Brick, P. (1996). Structure, 4, 287-298.

Cusack, S. (1997). Curr. Opin. Struct. Biol. 7, 881-889.

D'Ambrosio, H. K. \& Derbyshire, E. R. (2020). ACS Chem. Biol. 15, $17-27$.

Day, J. C., Goodall, T. I. \& Bailey, M. J. (2009). Mol. Phylogenet. Evol. 50, 93-101.

Duckworth, B. P., Nelson, K. M. \& Aldrich, C. C. (2012). Curr. Top. Med. Chem. 12, 766-796.

Emsley, P., Lohkamp, B., Scott, W. G. \& Cowtan, K. (2010). Acta Cryst. D66, 486-501.

Evans, P. R. \& Murshudov, G. N. (2013). Acta Cryst. D69, 1204-1214.

Fan, M., Xiao, Y., Li, M. \& Chang, W. (2016). Mol. Plant. 9, 13491352.

Gulick, A. M. (2009). ACS Chem. Biol. 4, 811-827.

Hisanaga, Y., Ago, H., Nakagawa, N., Hamada, K., Ida, K., Yamamoto, M., Hori, T., Arii, Y., Sugahara, M., Kuramitsu, S., Yokoyama, S. \& Miyano, M. (2004). J. Biol. Chem. 279, 3171731726.

Holm, L. \& Laakso, L. M. (2016). Nucleic Acids Res. 44, W351W355.

Huang, W., Xu, H., Li, Y., Zhang, F., Chen, X.-Y., He, Q.-L., Igarashi, Y. \& Tang, G.-L. (2012). J. Am. Chem. Soc. 134, 8831-8840.

Jones, D. T., Taylor, W. R. \& Thornton, J. M. (1992). Bioinformatics, 8 , 275-282.

Kabsch, W. (2010). Acta Cryst. D66, 125-132.

Krissinel, E. \& Henrick, K. (2007). J. Mol. Biol. 372, 774-797.

Kudo, F., Miyanaga, A. \& Eguchi, T. (2019). J. Ind. Microbiol. Biotechnol. 46, 515-536. 
Kumar, S., Stecher, G., Li, M., Knyaz, C. \& Tamura, K. (2018). Mol. Biol. Evol. 35, 1547-1549.

Labby, K. J., Watsula, S. G. \& Garneau-Tsodikova, S. (2015). Nat. Prod. Rep. 32, 641-653.

Laskowski, R. A., Jabłońska, J., Pravda, L., Vařeková, R. S. \& Thornton, J. M. (2018). Protein Sci. 27, 129-134.

Laskowski, R. A. \& Swindells, M. B. (2011). J. Chem. Inf. Model. 51, 2778-2786.

Law, A. \& Boulanger, M. J. (2011). J. Biol. Chem. 286, 15577-15585.

Liebschner, D., Afonine, P. V., Baker, M. L., Bunkóczi, G., Chen, V. B., Croll, T. I., Hintze, B., Hung, L.-W., Jain, S., McCoy, A. J., Moriarty, N. W., Oeffner, R. D., Poon, B. K., Prisant, M. G., Read, R. J., Richardson, J. S., Richardson, D. C., Sammito, M. D., Sobolev, O. V., Stockwell, D. H., Terwilliger, T. C., Urzhumtsev, A. G., Videau, L. L., Williams, C. J. \& Adams, P. D. (2019). Acta Cryst. D75, 861-877.

Losada, A. A., Cano-Prieto, C., García-Salcedo, R., Braña, A. F., Méndez, C., Salas, J. A. \& Olano, C. (2017). Microb. Biotechnol. 10, 873-885.

Lux, M. C., Standke, L. C. \& Tan, D. S. (2019). J. Antibiot. 72, 325-349.

Lv, M., Zhao, J., Deng, Z. \& Yu, Y. (2015). Chem. Biol. 22, 1313-1324.

Marahiel, M. A., Stachelhaus, T. \& Mootz, H. D. (1997). Chem. Rev. 97, 2651-2674.

McCoy, A. J. (2007). Acta Cryst. D63, 32-41.

Nakatsu, T., Ichiyama, S., Hiratake, J., Saldanha, A., Kobashi, N., Sakata, K. \& Kato, H. (2006). Nature, 440, 372-376.

Reger, A. S., Wu, R., Dunaway-Mariano, D. \& Gulick, A. M. (2008). Biochemistry, 47, 8016-8025.

Sato, S., Kajiura, T., Noguchi, M., Takehana, K., Kobayashi, T. \& Tsuji, T. (2001). J. Antibiot. 54, 102-104.
Schiering, N., Casale, E., Caccia, P., Giordano, P. \& Battistini, C. (2000). Biochemistry, 39, 13376-13382.

Schmelz, S. \& Naismith, J. H. (2009). Curr. Opin. Struct. Biol. 19, 666671.

Shao, X., Cao, H.-Y., Zhao, F., Peng, M., Wang, P., Li, C.-Y., Shi, W.-L., Wei, T.-D., Yuan, Z., Zhang, X.-H., Chen, X.-L., Todd, J. D. \& Zhang, Y.-Z. (2019). Mol. Microbiol. 111, 1057-1073.

Sievers, F., Wilm, A., Dineen, D., Gibson, T. J., Karplus, K., Li, W., Lopez, R., McWilliam, H., Remmert, M., Söding, J., Thompson, J. D. \& Higgins, D. G. (2011). Mol. Syst. Biol. 7, 539.

Song, H., Rao, C., Deng, Z., Yu, Y. \& Naismith, J. H. (2020). Angew. Chem. Int. Ed. 59, 6054-6061.

Stanišić, A. \& Kries, H. (2019). ChemBioChem, 20, 1347-1356.

Waterhouse, A. M., Procter, J. B., Martin, D. M., Clamp, M. \& Barton, G. J. (2009). Bioinformatics, 25, 1189-1191.

Winn, M. D., Ballard, C. C., Cowtan, K. D., Dodson, E. J., Emsley, P., Evans, P. R., Keegan, R. M., Krissinel, E. B., Leslie, A. G. W., McCoy, A., McNicholas, S. J., Murshudov, G. N., Pannu, N. S., Potterton, E. A., Powell, H. R., Read, R. J., Vagin, A. \& Wilson, K. S. (2011). Acta Cryst. D67, 235-242.

Wu, Q., Liang, J., Lin, S., Zhou, X., Bai, L., Deng, Z. \& Wang, Z. (2011). Antimicrob. Agents Chemother. 55, 974-982.

Yang, S., Cho, S. S., Levy, Y., Cheung, M. S., Levine, H., Wolynes, P. G. \& Onuchic, J. N. (2004). Proc. Natl Acad. Sci. USA, 101, 1378613791.

Zahn, M., Kurteva-Yaneva, N., Schuster, J., Krug, U., Georgi, T., Müller, R. H., Rohwerder, T. \& Sträter, N. (2019). J. Mol. Biol. 431, 2747-2761.

Ziemert, N. \& Jensen, P. R. (2012). Methods Enzymol. 517, 161182. 